\title{
Can Panax Ginseng Aqueous Extract Improve Chilled and Cryopreserved Bull Spermatozoa?
}

\author{
Falah Baiee ${ }^{1,2}$, Abd Wahid Haron ${ }^{2}$, Murtadha A. AL-mudhafr ${ }^{4}$, Innocent Damudu Peter ${ }^{2,3} \&$ Nurhusien Yimer ${ }^{2}$ \\ ${ }^{1}$ Department of Clinical Science, Faculty of Veterinary Medicine, University of Kufa, Najaf, Iraq \\ ${ }^{2}$ Department of Veterinary Clinical Studies, Faculty of Veterinary Medicine, Universiti Putra Malaysia, UPM \\ Serdang, Selangor, Malaysia \\ ${ }^{3}$ Faculty of Veterinary Medicine, University of Maiduguri, Maiduguri, Nigeria \\ ${ }^{4}$ Department of Microbiology, Faculty of Veterinary Medicine, University of Kufa, Najaf, Iraq \\ Correspondence: Falah Baiee, Department of Clinical Science, Faculty of Veterinary Medicine, University of Kufa, \\ 54003, Najaf, Iraq. Tel: 964-773-808-9998. E-mail: falahhali@uokufa.edu.iq
}

Received: May 29, 2020 Accepted: June 28, 2020 Online Published: July 14, 2020

The research is financed by (University Putra Malaysia, UPM researching grant; GPIPS/2016/9492800)

\begin{abstract}
This study was to evaluate the influence of Panax ginseng aqueous extract on chilled and frozen-thawed bull sperm quality. Samples of semen were acquired from four bulls through the use of an electro-ejaculator. Extension of the semen was done with tris-egg yolk diluent which was augmented with $0.0,0.25,0.5,1.0,2.5,5.0, \mathrm{and} 7.5 \mathrm{mg} / \mathrm{mL}$ Panax ginseng aqueous extract. Diluted chilled portions of the semen were chilled for 6 days at $5^{\circ} \mathrm{C}$ whereas the frozen semen was cryopreserved in liquid nitrogen. Results revealed that in chilled and frozen-thawed semen, the control group, $\mathrm{T}_{1}$ and $\mathrm{T}_{2}$ recorded higher percentages in terms of sperm motility and viability in all three groups evaluated compared to others, while the high dose of Panax ginseng aqueous extract in $\mathrm{T}_{6}$ and $\mathrm{T}_{5}$ recorded the lowest percentage. Moreover, the values of sperm morphology for chilled and frozen-thawed semen were not significant among the groups. The results of chromatin stability of the present study showed that $\mathrm{T}_{2}$ and control were higher than for other groups. In conclusion, the low dosage groups $\left(T_{1}, T 2\right.$ and $\left.T_{3}\right)$ which were received $(0.25$ $\mathrm{mg} / \mathrm{mL}, 0.5 \mathrm{mg} / \mathrm{mL}$ and $1 \mathrm{mg} / \mathrm{mL}$, respectively) from Panax ginseng aqueous extract were not significant as compared with the control group while high-dosage groups $\left(\mathrm{T}_{4}, \mathrm{~T}_{5}\right.$ and $\left.\mathrm{T}_{6}\right)$ which were received $(2.5 \mathrm{mg} / \mathrm{mL}, 5$ $\mathrm{mg} / \mathrm{mL}$ and $7.5 \mathrm{mg} / \mathrm{mL}$, respectively) from Panax ginseng aqueous extract were highly decreased spermatozoa characteristics.
\end{abstract}

Keywords: bull, semen cryopreservation, chilled semen, Panax ginseng, chromatin stability

\section{Introduction}

\subsection{Introduce the Problem}

Bovine insemination using the artificial insemination (AI) technique is among the main difficulties in the livestock industry. Semen preserved in liquid or frozen form are common procedures in inseminating cows; roughly $95 \%$ of AI dosages are in the form of frozen semen straws (Amann and Waberski, 2014). Nevertheless, freezing-thawing practices of prolonged semen are accountable for a substantial decrease in motility, viability, membrane integrity and morphology of sperm (Amirat-Briand et al., 2010; Yimer et al., 2014; Baiee et al., 2018a) resulting in decreased rate of fertility of the frozen semen (Büyükleblebici et al., 2014). In actual fact, chilled semen possesses a high rate of fertility in comparison to frozen semen (Medeiros et al., 2002; Galarza 2019); nonetheless, chilled semen can be kept for a few days only (Papa et al., 2015). Consequently, the use of chilled semen in the AI technique is restricted (Borges-Silva et al., 2016). Therefore, one purpose of this study was to determine the ability of a natural material in promoting chilled seminal quality for long-time storage and usage. Many studies have been done to improve frozen-thawed seminal quality through addition of some additives to the extended semen quality, for example, anti-oxidant (Amirat-Briand et al., 2010; Khumran et al., 2015; Eidan, 2016), or amino acids (Holt et al., 2015; Kumar et al., 2015) and unsaturated fatty acid (Kaka et al., 2015; Khoshvaght et al., 2015; Tarig et al., 2017). Furthermore, certain extracts of plants have been revealed as anti-oxidant additives which are used to add to semen or as a semen diluent (Chen et al., 1998; Baghshahi et al., 2014; Sapanidou et al., 2015), and to examine the toxic 
consequence of plant extract on sperm (Kaefer et al., 2013). Plant extracts are also used in diluting semen (Abul Rashid and Nurin, 2015). Based on our understanding, there are no studies that use Panax ginseng aqueous extract (PGe) in the tris-egg yolk diluent as enhancement for chilled and freezing semen.

Sperm present with Chromatin deficiencies, largely due to imperfect spermatogenesis, faulty apoptosis before ejaculation (Baiee et al., 2018b), extreme reactive oxygen species (ROS) production in the ejaculate (Moustafa et al., 2004), in vitro handling (Bollwein et al., 2008), and also the stress of the freezing-thawing processes (Khalifa et al., 2008; Lymberopoulos and Khalifa, 2010). In fact, frozen-thawed semen processes lead to chromatin damage (Métayer, et al., 2002; Gadea et al., 2008; Büyükleblebici et al., 2014). However, the mechanisms accountable for freezing-induced chromatin damage are unidentified. Data suggests that this could be the consequence of lipid peroxidation (LPO) in injurious sperm chromatin (Kasimanickam et al., 2007). Moreover, sperm nuclei exposure to high ionic strength in the course of freezing-thawing of semen instigates deterioration of chromatin assembly and sequentially, makes post-thawing nuclear DNA available to the oxidative occurrence through extra- or intracellular ROS (Gadea et al., 2008). Additionally, perhaps, the unrestrained post-thawing influx of wandering calcium ions in frozen sperm (Büyükleblebici et al., 2014) may possibly encourage additional splitting of nucleoprotein and Chromatin through endogenous protease and nuclease stimulation (Métayer et al., 2002).

Panax ginseng Meyer, from the family Araliaceae, is one of the most widely used traditional herbal medicines in Eastern and Western countries. Panax ginseng Meyer has been used in traditional medicine for over 2000 years to restore and enhance normal well-being, sexuality, promotion of vitality, erectile dysfunction, control of hypertension, and increase of resistance to stress and ageing (Attele et al., 1999; Kopalli et al., 2016). Active compounds in Panax ginseng include saponin (ginsenosides), acidic polysaccharides, peptides, polyacetylenes, alkaloids, and phenolic compounds (Attele et al., 1999). Ginsenosides of saponins are regarded as the main active components responsible for the pharmacological activities of Panax ginseng and approximately 40 kinds of saponin have been isolated from it. Most of these are protopanaxdiol and protopanaxtriol, which are aglycones of dammarane-type triterpenoids (Patel and Rauf 2017). Recently, physicochemical and spectroscopic data were acquired from four major diol-saponins: ginsenosides Rb1, Rb2, Rc, Re, Ro, and Rd (Cho et al., 2010). Thus, the main aim of this study was to determine and evaluate the effect of Panax ginseng aqueous extract on the quality of frozen-thawed bull semen. Furthermore, it was to determine the effect of Panax ginseng aqueous extract on chromatin stability of frozen-thawed bull semen.

\section{Method}

\subsection{Animals}

This work experiment was approved by the Institutional Animal Care and Use Committee, Faculty of Veterinary Medicine, UPM (RO73/2015). Twenty-four ejaculates were obtained through the use of the electro-ejaculator (EE; Ideal ${ }^{\circledR}$ Instruments; Neogen Corporation, Lansing, Michigan; USA) from four fertile crossbred (SimmentalBrangus) bulls from the Universiti Putra Malaysia (UPM) in Serdang, Selangor (2॰9-18.36-N; 101 $433-49.61-E)$ farm. Bulls were $4.8 \pm 0.8$ years old measuring $551.2 \pm 41.5 \mathrm{~kg}$ in weight. The entire four bulls were under the same feeding; the animals were fed with Brachiaria decumbens grass and also commercial palm kernel cake at 3 $\mathrm{kg} /$ body weight rate. Additionally, mineral blocks and water were given to the animals ad libitum.

\subsection{Chemicals}

Panax ginseng aqueous extract (GN06032014) was obtained from LJack (M)® Sdn. Bhd. Semenyih, Selangor, Malaysia with certification of the analysis. Tris, citric acid, sodium citrate, fructose, nigrosin, glycerol and eosiny were acquired from Sigma Aldrich Co. (Sigma, St. Louis, MO, USA). Penicillin-streptomycin was procured from Gibco ${ }^{\circledR}\left(15140122\right.$, Gibco ${ }^{\circledR}$ USA). Acridine orange stain was bought from Invitrogen ${ }^{\circledR}$ (AR7601000) Eugene; Oregon, USA; the straws were acquired from Minitube ${ }^{\circledR}(13407 / 0010$; Slovakia). Lastly, methanol and glacial acetic acid were obtained from Merck (E, Merck D6100 Darmstadt, and F. R. Germany).

\subsection{Preparation of Semen Diluent}

Preparation of Tris-egg yolk diluent was as earlier described by Amirat-Briand et al. (2010) with a slight adjustment (Baiee et al., 2017). Tris-egg yolk diluent was divided into two portions; the first portion (P1) contained Tris: 2.24 g, citric acid: $1.48 \mathrm{~g}$, fructose: $1 \mathrm{~g}$, $50000 \mathrm{IU}$ penicillin-streptomycin, and $20 \%$ egg yolk in $100 \mathrm{~mL}$ distilled water. The second portion (P2) had similar constituents to the initial but with $12.8 \%$ glycerol, the double amount of glycerol being to create the concluding concentration of glycerol after mixing with P1 6.4\%. Each portion was divided into seven parts in test tubes to give diverse concentrations of the extract of Panax ginseng. Furthermore, the diluents were weekly preparations and were kept at $-20^{\circ} \mathrm{C}$. 


\subsection{Semen Collection}

The collection of semen samples was done weekly very early in the morning through the use of auto mode of the EE. Bulls were directed to a chute. Prepuce hairs of the bulls were shaved; the preputial orifice was cleaned using water and dried with a clean tissue towel. Before insertion of rectal probe, rectal evacuation was done, and the EE device was switched on; a minimum of three individuals worked together to achieve semen sample collection. After sample collection, samples were conveyed to the Theriogenology and Cytogenetics lab at $37^{\circ} \mathrm{C}$. Computer assisted sperm analysis (CASA; Hamilton Thorne Bioscience; USA) was used to evaluate the concentration of semen and the proportion of sperm motility for fresh semen in addition to the universal sperm motility of chilled and cryopreserved semen. Semen samples with general sperm motility $\geq 70 \%$, sperm viability and normal sperm morphology $\geq 85 \%$ and concentration of $\geq 600 \times 10^{6}$ sperm $/ \mathrm{mL}$ (Kaka et al., 2015) were acknowledged to be chilled and cryopreserved

\subsection{Experimental Design}

The dosages of PGe were determined into six groups for all chilled and frozen-thawed experiments. Three groups with low concentrations were $T_{1} 0.25 \mathrm{mg} / \mathrm{mL}, T_{2} 0.5 \mathrm{mg} / \mathrm{mL}, T_{3} 1 \mathrm{mg} / \mathrm{mL}$, and three groups with high concentrations were $\mathrm{T}_{4} 2.5 \mathrm{mg} / \mathrm{mL}, \mathrm{T}_{5} 5 \mathrm{mg} / \mathrm{mL}, \mathrm{T}_{6} 7.5 \mathrm{mg} / \mathrm{mL}$. Moreover, one group without addition of PGe was kept to be a control group. The extract was dissolved into tris-egg yolk diluent by shaking and incubation in water path at $37^{\circ} \mathrm{C}$ for $10 \mathrm{~min}$. The successfully collected semen sample was divided into two equal aliquots, the 1 st aliquot was for the chilled semen experiment, and the 2nd aliquot was for the cryopreserved experiment.

\subsubsection{Effect of Panax Ginseng on Chilled Bull Seminal Quality}

The first aliquot of fresh semen was equally divided into seven groups which contained diverse concentrations of $\mathrm{PGe}$ which were dissolved in tris-egg yolk diluent (P1, deprived of glycerol). Extended semen groups were store in a refrigerator for six days at $5^{\circ} \mathrm{C}$. Sperm morphology, viability and motility were assessed after 1,3 and 6 days for cooling. Furthermore, sperm membrane integrity was assessed at day three of chilling in the entire groups. The final concentration of semen in chilled experiments was about $80 \times 10^{6} \mathrm{sperm} / \mathrm{mL}$.

\subsubsection{Effect of Panax Ginseng on Frozen-Thawed Bull Seminal Quality}

The second aliquot of the fresh semen was also equally divided into seven groups which contained tris-egg yolk diluent augmented with diverse concentrations of PGe (P1), they were chilled in a refrigerator at $5^{\circ} \mathrm{C}$ for three hours. The second portion of diluent which contained glycerol (P2) was added to the entire treatment groups; it was later packaged in $0.25 \mathrm{~mL}$ French straws. The end concentration of glycerol in the diluent was $6.4 \%$ while the end concentration of spermatozoa was $20 \times 10^{6} \mathrm{sperm} /$ straw. The entire groups of straws were frozen at liquid nitrogen for future investigation. Motility of sperm, morphology and viability values were recorded in 2, 7 and 14 days after freezing. Sperm membrane integrity was recorded after 7 days, and sperm DNA integrity was also recorded after 20 days of freezing.

\subsection{Evaluation of Sperm Motility, Morphology and Viability}

Sperm viability and normal morphology proportions were investigated through the use of eosin-nigrosin stain (EN) as was earlier described by Felipe-Pérez et al. (2008). Briefly, a $10 \mu \mathrm{L}$ droplet of freshly chilled or cryopreserved semen was added with $20 \mu \mathrm{L}$ of EN stain; the mixture was smeared on a microscope slide and kept to dry at $45^{\circ} \mathrm{C}$ on a slide warmer. Subsequently the slides were viewed under a phase-contrast microscope (Nikon Japan, Eclipse E200) at $\times 400$ for viability of the sperm. Sperm that were stained with pink colour were considered to be dead while the unstained sperm were deemed to be alive. Additionally, in the usual sperm morphology, only sperm with normal sperm head, midpiece and tail are referred to as normal irrespective of the colour at $\times 1000$ with oil emersion, and for each test 200 sperm were counted.

\subsection{Evaluation of Sperm Membrane Reliability}

The hypo-osmotic swelling test (HOST) was done to investigate the sperm membrane reliability in chilled and cryopreserved sperm. Essentially, HOST was prepared as was earlier described by Revell and Mrode (1994). Briefly, $0.9 \mathrm{~g}$ fructose and $0.49 \mathrm{~g}$ tri-sodium citrate were dissolved in distilled water; additional distilled water was added to make up the volume to $100 \mathrm{~mL}$. Ten microliters of chilled or cryopreserved sperm were incubated with $90 \mu \mathrm{L}$ of hypo-osmotic solution for $30 \mathrm{~min}$ at $37^{\circ} \mathrm{C}$. Subsequently $10 \mu \mathrm{L}$ of incubated solution was positioned on a glass slide and covered using a cover slip and observed under the phase-contrast microscope at $\times 400$. Sperm possessing twisted tails under hypo-osmotic conditions were considered to possess complete membranes.

\subsection{Evaluation of Sperm Chromatin Stability}

Acridine orange test (AOT) was performed in determining sperm chromatin stability in cryopreserved sperm as 
was described by Tejada et al. (1984). A metachromatic dye, acridine orange (AO) stain interpolates the DNA of sperm cells to release colours fluctuating between green, green yellow, yellow, orange, and red. Sperm cells with intact chromatin radiated green fluorescence, whereas those possessing altered chromatin radiated green yellow, yellow, orange, and red (Figure 1) dependent on the bulk of fragmented chromatin. Consequently, the outcomes were divided into five groupings based on the percentage of chromatin destroyed. A thick semen smear was made on a glass slide and kept to dry. Carnoy's solution was daily prepared for the smear fixing for 3 hours at a chilled temperature. The slides were allowed to dry once more and stained with AO staining solution for 5 minutes; the slides were then washed gently using distilled water. Slides were allowed to dry and were covered using cover slips and observed under a fluorescence microscope (Nikon eclipse, Ti-s Japan) within 30 minutes. This is because this test ought to be done within 30 a minimum of three microscopic fielded positions were taken from each slide for each group and saved in the computer for further evaluation.
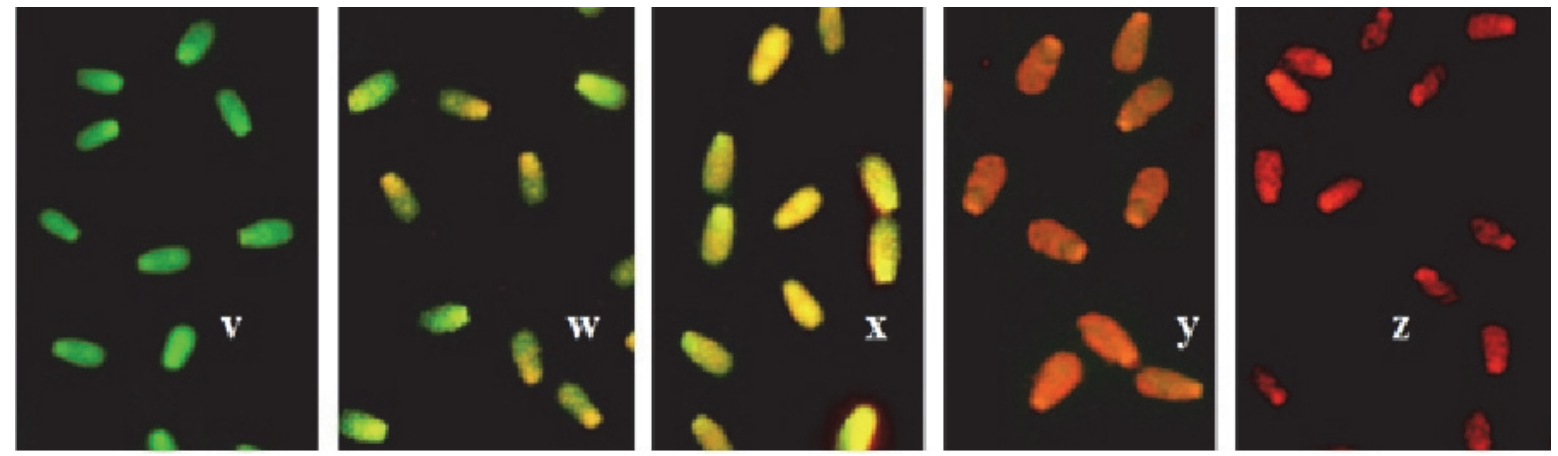

Figure 1. v, w, x, y, and z. Examples of spermatozoa are from bulls stained with Acridine orange stain and examined under fluorescence microscope (Nikon eclipse, Ti-s Japan). Acridine orange stain emits green colour under blue fluorescence laser meaning to normal chromatin; but, it releases green-yellow, yellow, orange, and red meaning to chromatin impairment.

v: shows a green sperm that had relatively intact chromatin

w: green-yellow sperm that has $75 \%$ intact chromatin

$\mathrm{x}$ : Yellow sperm that has $50 \%$ intact chromatin

$y$ : orange sperm that has $25 \%$ intact chromatin

$\mathrm{z}$ : red sperm head that has relatively total chromatin impairment

\subsection{Statistical Analysis}

Data obtained were tested for normal distribution through the use of the Shapiro-wilk test. Two-way ANOVA was utilized in comparing sperm motility, viability and morphology amongst different groups and different evaluation times while one-way ANOVA was used in comparing sperm membrane integrity and chromatin integrity among groups. Two-way and one-way ANOVA were followed by the LSD test in comparing the significances among groups through the use of the SPSS programme version 22 for windows (SPSS Inc., Chicago, IL; the USA).

\section{Results}

The values of chilled semen for sperm motility (Table 1), morphology (Table 2) and viability (Table 3 ) were evaluated at three different times. Control, T1 and T2 groups recorded the highest percentage in terms of sperm motility and viability in all three evaluations as compared among groups for each time of evaluation, while the high dose of PGe in T5 and T6 recorded the lowest percentage. Moreover, the mean percentages of sperm morphology for chilled semen were not significant among groups. On the other hand, there are significant differences in terms of time of evaluation, since the values were significantly different for the first evaluation as compared to the third chilled semen evaluation. Sperm morphology values were also decreased during time of preservation in chilled form, but it was not significantly different among periods. 
Table 1. Effect of Panax ginseng aqueous extract on chilled semen motility in three different times of evaluation

\begin{tabular}{|c|c|c|c|c|}
\hline \multirow[b]{2}{*}{ Parameters } & \multirow[b]{2}{*}{ Groups } & \multicolumn{3}{|l|}{ Mean \pm SEM } \\
\hline & & $\begin{array}{l}1^{\text {st }} \text { Evaluation } \\
1 \mathrm{~d}\end{array}$ & $\begin{array}{l}2^{\text {nd }} \text { Evaluation } \\
3 \mathrm{~d}\end{array}$ & $\begin{array}{l}3^{\text {rd }} \text { Evaluation } \\
6 \mathrm{~d}\end{array}$ \\
\hline $\begin{array}{l}\text { Sperm motility } \\
(\%)\end{array}$ & $\begin{array}{l}\mathrm{T}_{1} \\
\mathrm{~T}_{2} \\
\mathrm{~T}_{3} \\
\mathrm{~T}_{4} \\
\mathrm{~T}_{5} \\
\mathrm{~T}_{6} \\
\text { Control }\end{array}$ & $\begin{array}{l}75.69 \pm 1.84^{\mathrm{aX}} \\
79.94 \pm 1.16^{\mathrm{aX}} \\
66.44 \pm 1.77^{\mathrm{bX}} \\
44.19 \pm 3.13^{\mathrm{cX}} \\
13.13 \pm 1.67^{\mathrm{cdX}} \\
4.94 \pm 0.71^{\mathrm{d}} \\
75.00 \pm 2.06^{\mathrm{aX}}\end{array}$ & $\begin{array}{l}61.62 \pm 3.27^{\mathrm{aY}} \\
64.87 \pm 2.35^{\mathrm{aY}} \\
52.25 \pm 2.97^{\mathrm{bX}} \\
22.62 \pm 3.49^{\mathrm{cY}} \\
3.94 \pm 0.91^{\mathrm{dXY}} \\
0.41 \pm 0.11^{\mathrm{d}} \\
59.56 \pm 2.31^{\mathrm{aY}}\end{array}$ & $\begin{array}{l}28.19 \pm 2.04^{\mathrm{aZ}} \\
28.88 \pm 2.02^{\mathrm{aZ}} \\
17.13 \pm 1.62^{\mathrm{bY}} \\
3.25 \pm 1.02^{\mathrm{cZ}} \\
0.70 \pm 0.32^{\mathrm{cY}} \\
0.00 \pm 0.00^{\mathrm{c}} \\
27.12 \pm 2.45^{\mathrm{aZ}}\end{array}$ \\
\hline
\end{tabular}

$\mathrm{T}_{1}=0.25 \mathrm{mg} / \mathrm{mL}, \mathrm{T}_{2}=0.5 \mathrm{mg} / \mathrm{mL}, \mathrm{T}_{3}=1 \mathrm{mg} / \mathrm{mL}, \mathrm{T}_{4}=2.5 \mathrm{mg} / \mathrm{mL}, \mathrm{T}_{5}=5 \mathrm{mg} / \mathrm{mL}, \mathrm{T}_{6} 7.5 \mathrm{mg} / \mathrm{mL}$, and Control $=$ $0.0 \mathrm{mg} / \mathrm{mL}$

$\mathrm{n}=24$

a, b,c, \& d superscripts mean between groups were significantly different at $(\mathrm{P}<0.05$; within column between groups). $\mathrm{x}, \mathrm{y} \& \mathrm{z}$ superscripts mean between times of evaluation were significantly different at $(\mathrm{P}<0.01$; between columns for the same group).

Table 2. Effect of Panax ginseng aqueous extract on chilled semen normal morphology in three different times of evaluation

\begin{tabular}{llccc}
\hline \multirow{2}{*}{ Parameters } & Groups & \multicolumn{3}{c}{ Mean \pm SEM } \\
\cline { 3 - 5 } & & $1^{\text {st }}$ Evaluation & $2^{\text {nd }}$ Evaluation & $3^{\text {rd }} \begin{array}{c}\text { Evaluation } \\
6 \mathrm{~d}\end{array}$ \\
\hline & $\mathrm{T}_{1}$ & $89.44 \pm 0.66$ & $88.47 \pm 0.77$ & $86.94 \pm 1.07$ \\
& $\mathrm{~T}_{2}$ & $89.66 \pm 0.79$ & $88.12 \pm 0.89$ & $87.19 \pm 0.93$ \\
Sperm & $\mathrm{T}_{3}$ & $87.47 \pm 0.83$ & $86.59 \pm 0.99$ & $86.28 \pm 0.86$ \\
morphology & $\mathrm{T}_{4}$ & $88.44 \pm 0.97$ & $88.03 \pm 0.91$ & $86.06 \pm 1.10$ \\
$(\%)$ & $\mathrm{T}_{5}$ & $87.69 \pm 0.93$ & $86.75 \pm 0.64$ & $86.13 \pm 1.01$ \\
& $\mathrm{~T}_{6}$ & $88.06 \pm 0.96$ & $87.03 \pm 0.92$ & $86.19 \pm 1.11$ \\
& Control & $89.19 \pm 1.00$ & $88.25 \pm 0.93$ & $87.31 \pm 1.22$ \\
\hline
\end{tabular}

$\mathrm{T}_{1}=0.25 \mathrm{mg} / \mathrm{mL}, \mathrm{T}_{2}=0.5 \mathrm{mg} / \mathrm{mL}, \mathrm{T}_{3}=1 \mathrm{mg} / \mathrm{mL}, \mathrm{T}_{4}=2.5 \mathrm{mg} / \mathrm{mL}, \mathrm{T}_{5}=5 \mathrm{mg} / \mathrm{mL}, \mathrm{T}_{6} 7.5 \mathrm{mg} / \mathrm{mL}$, and Control $=$ $0.0 \mathrm{mg} / \mathrm{mL}$

$\mathrm{n}=24$

Table 3. Effect of Panax ginseng aqueous extract on chilled semen viability in three different times of evaluation

\begin{tabular}{|c|c|c|c|c|}
\hline \multirow[b]{2}{*}{ Parameters } & \multirow[b]{2}{*}{ Groups } & \multicolumn{3}{|l|}{ Mean \pm SEM } \\
\hline & & $\begin{array}{l}1^{\text {st }} \text { Evaluation } \\
1 \mathrm{~d}\end{array}$ & $\begin{array}{l}2^{\text {nd }} \text { Evaluation } \\
3 \mathrm{~d}\end{array}$ & $\begin{array}{l}3^{\text {rd }} \text { Evaluation } \\
6 \mathrm{~d}\end{array}$ \\
\hline \multirow{7}{*}{$\begin{array}{l}\text { Sperm viability } \\
(\%)\end{array}$} & $\mathrm{T}_{1}$ & $78.72 \pm 1.45^{\mathrm{aX}}$ & $70.41 \pm 1.74^{\mathrm{aX}}$ & $52.10 \pm 2.21^{\mathrm{abY}}$ \\
\hline & $\mathrm{T}_{2}$ & $80.72 \pm 1.25^{\mathrm{aX}}$ & $71.78 \pm 1.60^{\mathrm{aX}}$ & $56.47 \pm 1.89^{\mathrm{aY}}$ \\
\hline & $\mathrm{T}_{3}$ & $67.75 \pm 2.39^{\mathrm{bX}}$ & $60.31 \pm 2.18^{\mathrm{bX}}$ & $49.06 \pm 2.42^{\mathrm{bY}}$ \\
\hline & $\mathrm{T}_{4}$ & $48.56 \pm 5.24^{\mathrm{cX}}$ & $41.15 \pm 4.58^{\mathrm{cXY}}$ & $33.41 \pm 3.69^{\mathrm{cY}}$ \\
\hline & $\mathrm{T}_{5}$ & $26.03 \pm 4.04^{\mathrm{cdX}}$ & $17.75 \pm 2.18^{\mathrm{cdXY}}$ & $12.00 \pm 1.79^{\mathrm{cdY}}$ \\
\hline & $\mathrm{T}_{6}$ & $16.25 \pm 2.68^{\mathrm{dX}}$ & $9.22 \pm 1.14^{\mathrm{dXY}}$ & $4.50 \pm 0.77^{\mathrm{dY}}$ \\
\hline & Control & $78.16 \pm 1.14^{\mathrm{aX}}$ & $67.22 \pm 1.21^{\mathrm{aX}}$ & $52.66 \pm 1.35^{\mathrm{abY}}$ \\
\hline
\end{tabular}

$\mathrm{T}_{1}=0.25 \mathrm{mg} / \mathrm{mL}, \mathrm{T}_{2}=0.5 \mathrm{mg} / \mathrm{mL}, \mathrm{T}_{3}=1 \mathrm{mg} / \mathrm{mL}, \mathrm{T}_{4}=2.5 \mathrm{mg} / \mathrm{mL}, \mathrm{T}_{5}=5 \mathrm{mg} / \mathrm{mL}, \mathrm{T}_{6} 7.5 \mathrm{mg} / \mathrm{mL}$, and Control $=$ $0.0 \mathrm{mg} / \mathrm{mL}$

$\mathrm{n}=24$

a, b,c, \& d superscripts mean between groups were significantly different at $(\mathrm{P}<0.05$; within column between groups).

${ }^{x, y} \& \mathrm{z}$ superscripts mean between times of evaluation were significantly different at $(\mathrm{P}<0.01$; between columns for the same group). 
Frozen-thawed semen was also evaluated into three different times in terms of sperm motility (Table 4), morphology (Table 5) and viability (Table 6). Control, T1 and T2 groups were significantly higher than other groups in terms of sperm motility and viability values for 1 st, 2 nd and 3 rd evaluations. The low percentage of sperm motility was recorded in high dose of $\mathrm{PGe} \mathrm{T} 6=7.5 \mathrm{mg} / \mathrm{mL}(0.00 \pm 0.00 \%)$ in all the three different periods, followed by $\mathrm{T} 5=5 \mathrm{mg} / \mathrm{mL}(0.31 \pm 0.10,0.25 \pm 0.10,0.28 \pm 0.09$, respectively). Moreover, sperm morphology values of frozen-thawed semen did not differ among groups which had different concentrations of PGe in all the three times of evaluations. On the other hand, there were no significant differences among 1st, 2nd and 3rd evaluations in terms of sperm motility, morphology and viability $(\mathrm{P}>0.05)$.

Table 4. Effect of Panax ginseng aqueous extract on frozen-thawed semen motility in three different times of evaluation

\begin{tabular}{lllll}
\hline \multirow{3}{*}{ Parameters } & Groups & \multicolumn{3}{l}{ Mean \pm SEM } \\
\cline { 2 - 4 } & & $\begin{array}{l}1^{\text {st }} \text { Evaluation } \\
2 \text { days }\end{array}$ & $\begin{array}{l}2^{\text {nd }} \text { Evaluation } \\
7 \text { days }\end{array}$ & $\begin{array}{l}3^{\text {rd }} \text { Evaluation } \\
14 \text { days }\end{array}$ \\
\hline \multirow{4}{*}{ Sperm motility } & $46.81 \pm 2.98^{\mathrm{a}}$ & $46.00 \pm 3.15^{\mathrm{a}}$ & $49.13 \pm 3.05^{\mathrm{a}}$ \\
$(\%)$ & $\mathrm{T}_{1}$ & $43.31 \pm 3.37^{\mathrm{a}}$ & $39.50 \pm 2.24^{\mathrm{a}}$ & $40.00 \pm 2.42^{\mathrm{a}}$ \\
& $\mathrm{T}_{2}$ & $15.38 \pm 1.05^{\mathrm{b}}$ & $16.06 \pm 2.09^{\mathrm{b}}$ & $16.68 \pm 2.05^{\mathrm{b}}$ \\
& $\mathrm{T}_{3}$ & $5.06 \pm 1.07^{\mathrm{c}}$ & $3.44 \pm 0.88^{\mathrm{c}}$ & $3.44 \pm 0.65^{\mathrm{c}}$ \\
& $\mathrm{T}_{4}$ & $0.31 \pm 0.10^{\mathrm{c}}$ & $0.25 \pm 0.10^{\mathrm{c}}$ & $0.28 \pm 0.09^{\mathrm{c}}$ \\
& $\mathrm{T}_{5}$ & $0.00 \pm 0.00^{\mathrm{c}}$ & $0.00 \pm 0.00^{\mathrm{c}}$ & $0.00 \pm 0.00^{\mathrm{c}}$ \\
& $\mathrm{T}_{6}$ & $46.94 \pm 2.19^{\mathrm{a}}$ & $48.87 \pm 3.41^{\mathrm{a}}$ & $51.25 \pm 2.76^{\mathrm{a}}$ \\
\hline
\end{tabular}

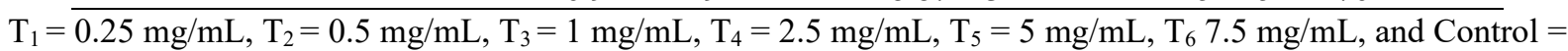
$0.0 \mathrm{mg} / \mathrm{mL}$

$\mathrm{n}=24$

$\mathrm{a}, \mathrm{b} \& \mathrm{c}$ superscripts mean between groups were significantly different at $(\mathrm{P}<0.05$; within column between groups).

Table 5. Effect of Panax ginseng aqueous extract on frozen-thawed semen morphology in three different times of evaluation

\begin{tabular}{lllll}
\hline \multirow{3}{*}{ Parameters } & Groups & \multicolumn{3}{l}{ Mean \pm SEM } \\
\cline { 3 - 5 } & & $\begin{array}{l}1^{\text {st }} \text { Evaluation } \\
2 \text { days }\end{array}$ & $\begin{array}{l}2^{\text {nd }} \text { Evaluation } \\
7 \text { days }\end{array}$ & $\begin{array}{l}3^{\text {rd }} \text { Evaluation } \\
14 \text { days }\end{array}$ \\
\hline & $\mathrm{T}_{1}$ & $90.91 \pm 0.67$ & $90.11 \pm 0.71$ & $90.25 \pm 0.63$ \\
& $\mathrm{~T}_{2}$ & $90.69 \pm 0.56$ & $90.69 \pm 0.59$ & $90.59 \pm 0.45$ \\
Sperm & $\mathrm{T}_{3}$ & $89.22 \pm 0.81$ & $89.47 \pm 0.73$ & $88.85 \pm 0.70$ \\
morphology & $\mathrm{T}_{4}$ & $88.91 \pm 0.80$ & $89.00 \pm 0.79$ & $89.13 \pm 0.75$ \\
$(\%)$ & $\mathrm{T}_{5}$ & $87.97 \pm 0.80$ & $88.25 \pm 0.86$ & $88.41 \pm 0.69$ \\
& $\mathrm{~T}_{6}$ & $88.19 \pm 0.74$ & $88.06 \pm 0.88$ & $87.69 \pm 0.77$ \\
& Control & $91.47 \pm 0.75$ & $91.19 \pm 0.66$ & $91.31 \pm 0.54$ \\
\hline
\end{tabular}

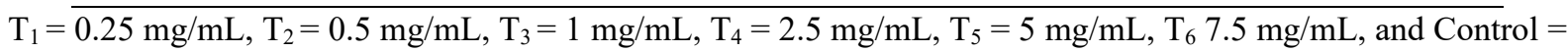
$0.0 \mathrm{mg} / \mathrm{mL}$

$\mathrm{n}=24$ 
Table 6. Effect of Panax ginseng aqueous extract on frozen-thawed semen viability in three different times of evaluation

\begin{tabular}{lllll}
\hline \multirow{2}{*}{ Parameters } & Groups & \multicolumn{3}{l}{ Mean \pm SEM } \\
\cline { 3 - 5 } & $\begin{array}{llll}1^{\text {st }} \text { Evaluation } \\
2 \text { days }\end{array}$ & $\begin{array}{l}2^{\text {nd }} \text { Evaluation } \\
7 \text { days }\end{array}$ & $\begin{array}{l}3^{\text {rd }} \\
14 \text { days }\end{array}$ \\
\hline \multirow{3}{*}{ Sperm viability } & $65.03 \pm 2.50^{\mathrm{a}}$ & $65.13 \pm 2.54^{\mathrm{a}}$ & $65.84 \pm 2.65^{\mathrm{a}}$ \\
$(\%)$ & $\mathrm{T}_{1}$ & $58.44 \pm 3.12^{\mathrm{a}}$ & $57.28 \pm 2.95^{\mathrm{a}}$ & $57.28 \pm 2.97^{\mathrm{a}}$ \\
& $\mathrm{T}_{2}$ & $31.97 \pm 2.72^{\mathrm{b}}$ & $32.56 \pm 2.75^{\mathrm{b}}$ & $33.28 \pm 0.95^{\mathrm{b}}$ \\
& $\mathrm{T}_{4}$ & $13.94 \pm 1.92^{\mathrm{c}}$ & $13.69 \pm 2.14^{\mathrm{c}}$ & $13.66 \pm 1.64^{\mathrm{c}}$ \\
& $\mathrm{T}_{5}$ & $6.91 \pm 0.90^{\mathrm{c}}$ & $6.09 \pm 0.73^{\mathrm{c}}$ & $6.13 \pm 0.72^{\mathrm{c}}$ \\
& $\mathrm{T}_{6}$ & $3.47 \pm 0.53^{\mathrm{c}}$ & $3.04 \pm 0.38^{\mathrm{c}}$ & $3.25 \pm 0.48^{\mathrm{c}}$ \\
& Control & $66.03 \pm 1.71^{\mathrm{a}}$ & $66.06 \pm 1.79^{\mathrm{a}}$ & $66.34 \pm 1.74^{\mathrm{a}}$
\end{tabular}

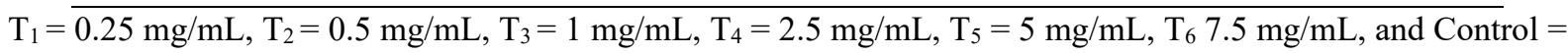
$0.0 \mathrm{mg} / \mathrm{mL}$

$\mathrm{n}=24$

a, $b$ \& c superscripts mean between groups were significantly different at $(\mathrm{P}<0.05$; within column between groups)

Sperm membrane reliability of chilled and frozen-thawed spermatozoa was evaluated using HOST. The collected data of chilled semen referred to as $\mathrm{T}_{1}(66.03 \pm 2.05 \%), \mathrm{T}_{2}(66.84 \pm 2.75 \%)$ and control $(65.03 \pm 1.52 \%)$ groups were higher significantly at ( $\square \square 0.01)$ as compared to $\mathrm{T}_{3}(53.62 \pm 3.89 \%), \mathrm{T}_{4}(36.84 \pm 4.89 \%), \mathrm{T}_{5}(14.78 \pm 2.58 \%)$, and $\mathrm{T}_{6}(7.25 \pm 1.39 \%)$ in chilled semen (Figure 2). Moreover, the collected data of frozen-thawed semen revealed that control $(58.28 \pm 2.13 \%)$, was higher than $\mathrm{T}_{1}(57.44 \pm 1.96 \% ; \mathrm{P}=0.73), \mathrm{T}_{2}(52.56 \pm 2.15 \% ; \mathrm{P} \square 0.05), \mathrm{T}_{3}$ $(29.31 \pm 2.17 \%), \mathrm{T}_{4}(13.12 \pm 1.54 \%), \mathrm{T}_{5}(5.97 \pm 0.92 \%)$, and $\mathrm{T}_{6}(3.31 \pm 0.54 \% ; \mathrm{P} \square 0.01)$ in terms of sperm membrane reliability (Figure 3$)$. There was no significant difference between $T_{1}$ and $T_{2}(P=0.06)$. In fact, the percentage of sperm membrane reliability decreased as the dosages increased in chilled and frozen-thawed bull semen.

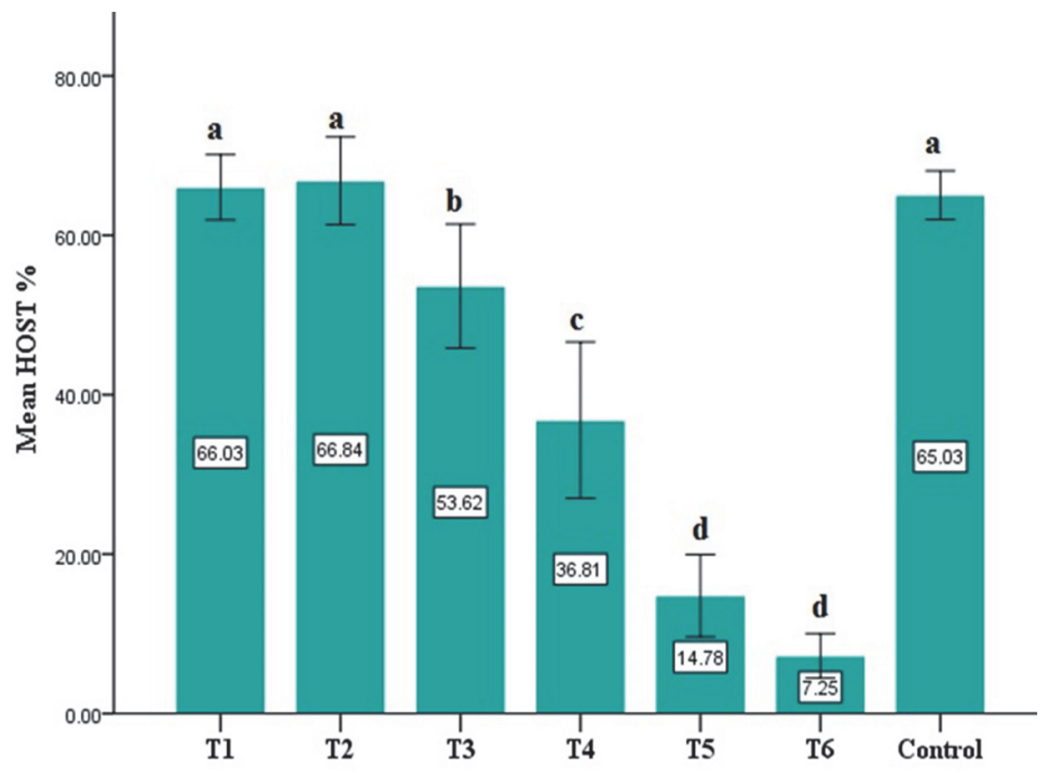

Figure 2. Effect of Panax ginseng extract on sperm membrane integrity of chilled bull sperm (\%; Mean $\pm \mathrm{SEM})$. $\mathrm{T}_{1}=0.25 \mathrm{mg} / \mathrm{mL}, \mathrm{T}_{2}=0.5 \mathrm{mg} / \mathrm{mL}, \mathrm{T}_{3}=1 \mathrm{mg} / \mathrm{mL}, \mathrm{T}_{4}=2.5 \mathrm{mg} / \mathrm{mL}, \mathrm{T}_{5}=5 \mathrm{mg} / \mathrm{mL}, \mathrm{T}_{6} 7.5 \mathrm{mg} / \mathrm{mL}$, and Control $=$ $0.0 \mathrm{mg} / \mathrm{mL}$

$\mathrm{n}=24$

a, b, c and d superscripts mean between groups were significantly different 


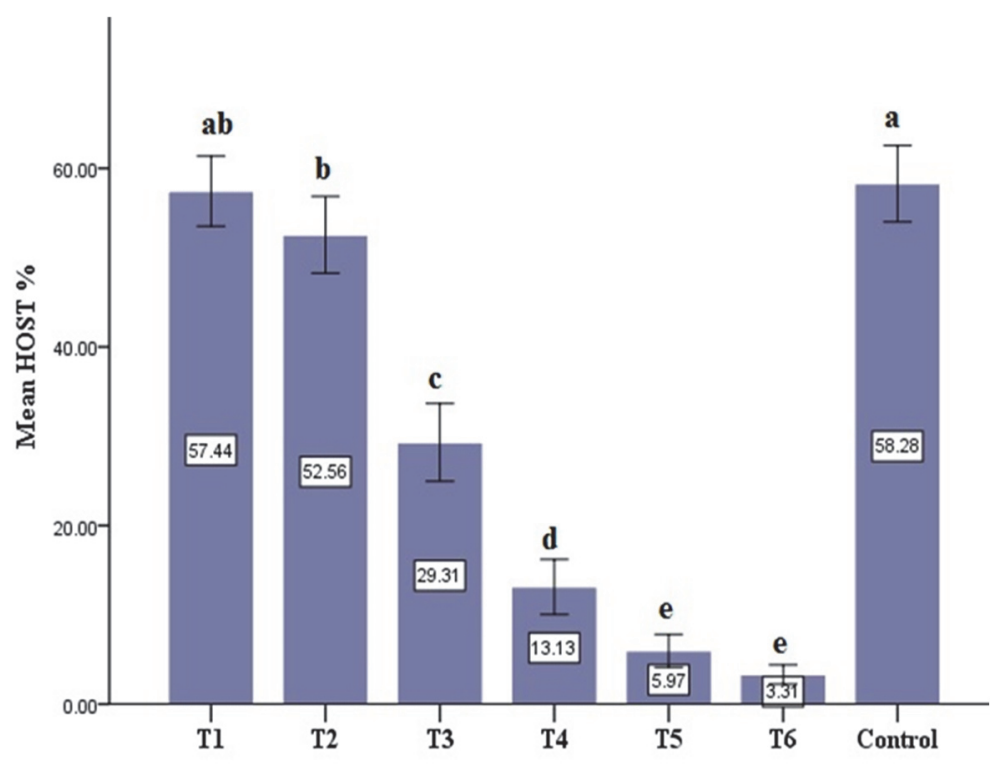

Figure 3. Effect of Panax ginseng extract on sperm membrane integrity of frozen-thawed bull sperm (\%; Mean \pm SEM)

$\mathrm{T}_{1}=0.25 \mathrm{mg} / \mathrm{mL}, \mathrm{T}_{2}=0.5 \mathrm{mg} / \mathrm{mL}, \mathrm{T}_{3}=1 \mathrm{mg} / \mathrm{mL}, \mathrm{T}_{4}=2.5 \mathrm{mg} / \mathrm{mL}, \mathrm{T}_{5}=5 \mathrm{mg} / \mathrm{mL}, \mathrm{T}_{6} 7.5 \mathrm{mg} / \mathrm{mL}$, and Control $=$ $0.0 \mathrm{mg} / \mathrm{mL}$

$\mathrm{n}=24$

a, b, c, d, and e superscripts mean between groups were significantly different.

Sperm chromatin integrity was evaluated using Acridine orange stain that was examined under a fluorescence microscope during $30 \mathrm{~min}$ after staining. Because the AO stain emits colour shifting from green to red, the calculated spermatozoa were distributed into five categories depending on the colour that emitted from them. In fact, only the green head of spermatozoa were considered to be entirely chromatin. The results of the present study showed that $\mathrm{T}_{2}(75.40 \pm 5.27)$ and control $(75.02 \pm 5.10)$ groups were significantly higher than other groups except $\mathrm{T}_{1}(71.15 \pm 4.93)$ which was not significant (Table 7). On the other hand, the chromatin impairment which situated under red was higher in $\mathrm{T}_{3}$ followed by $\mathrm{T}_{4}, \mathrm{~T}_{6}$ and $\mathrm{T}_{5}(15.09 \pm 2.33,13.95 \pm 1.26,12.26 \pm 3.51$ and $11.71 \pm 2.87$; respectively).

Table 7. Effect of Panax ginseng extract on chromatin stability of frozen-thawed bull sperm $(\% ; \mathrm{Mean} \pm \mathrm{SEM})$

\begin{tabular}{llllll}
\hline Groups & Green $^{*}$ & Green-yellow & Yellow & Orange & Red \\
\hline $\mathrm{T}_{1}$ & $71.15 \pm 4.93^{\mathrm{ab}}$ & $4.14 \pm 0.98$ & $8.82 \pm 1.96$ & $12.25 \pm 1.96$ & $5.79 \pm 2.22^{\mathrm{a}}$ \\
$\mathrm{T}_{2}$ & $75.40 \pm 5.27^{\mathrm{a}}$ & $3.69 \pm 0.99$ & $7.43 \pm 1.88$ & $9.20 \pm 2.40$ & $5.97 \pm 1.36^{\mathrm{a}}$ \\
$\mathrm{T}_{3}$ & $70.19 \pm 2.83$ & $3,41 \pm 0.94$ & $6.05 \pm 1.14$ & $6.67 \pm 2.12$ & $15.09 \pm 2.33^{\mathrm{b}}$ \\
$\mathrm{T}_{4}$ & $64.29 \pm 3.59^{\mathrm{b}}$ & $4.20 \pm 0.73$ & $5.01 \pm 1.58$ & $13.75 \pm 3.68$ & $13.95 \pm 1.26^{\mathrm{b}}$ \\
$\mathrm{T}_{5}$ & $59.48 \pm 2.90^{\mathrm{c}}$ & $5.73 \pm 1.05$ & $11.65 \pm 1.91$ & $14.16 \pm 3.34$ & $11.71 \pm 2.87^{\mathrm{b}}$ \\
$\mathrm{T}_{6}$ & $63.53 \pm 4.66^{\mathrm{c}}$ & $4.08 \pm 1.14$ & $9.40 \pm 1.57$ & $12.82 \pm 1.74$ & $12.26 \pm 3.51^{\mathrm{b}}$ \\
Control & $75.02 \pm 5.10^{\mathrm{a}}$ & $3.77 \pm 0.91$ & $7.85 \pm 2.19$ & $10.05 \pm 4.06$ & $5.08 \pm 1.69^{\mathrm{a}}$ \\
\hline
\end{tabular}

* Green: sperm had totally intact DNA

Green-yellow: sperm had 75\% intact DNA

Yellow: sperm had 50\% intact DNA

Orange: sperm had $25 \%$ intact DNA

Red: sperm that had totally damaged.

$\mathrm{T}_{1}=0.25 \mathrm{mg} / \mathrm{mL}, \mathrm{T}_{2}=0.5 \mathrm{mg} / \mathrm{mL}, \mathrm{T}_{3}=1 \mathrm{mg} / \mathrm{mL}, \mathrm{T}_{4}=2.5 \mathrm{mg} / \mathrm{mL}, \mathrm{T}_{5}=5 \mathrm{mg} / \mathrm{mL}, \mathrm{T}_{6} 7.5 \mathrm{mg} / \mathrm{mL}$, and Control = $0.0 \mathrm{mg} / \mathrm{mL}$.

$\mathrm{n}=24$

Different superscripts ${ }^{\mathrm{a}, \mathrm{b}}$ and ${ }^{\mathrm{c}}$ in the same column demonstrates statistical different among groups at $(\mathrm{P}<0.05)$ 


\section{Discussion}

Studies have shown that addition of plant origin additives such as clove bud (Baghshahi et al., 2014), curcumin (Tvrdá et al., 2016), soy-bean lecithin (Chelucci et al., 2015), virgin coconut oil (Tarig et al., 2017), and corcin (Sapanidou et al., 2015) might improve some characteristics of the chilled or frozen-thawed seminal quality. Panax ginseng is well demonstrated in literature to remove the harmful effect of ROS, to restore and enhance normal wellbeing, to promote vitality, and to increase resistance to stress (Attele et al., 1999; Kopalli et al., 2016a;b). To our knowledge, no study has been conducted to evaluate the effect of PGe on tris-egg yolk diluent to improve the quality of chilled and frozen-thawed bull semen. Therefore, one purpose of this study was to determine the ability of natural material in promoting chilled seminal quality for long-time storage and usage. Moreover, this study was to determine the effect of PGe supplemented in bull semen diluent that had been stored in frozen form. In the present study, six doses of PGe have been distributed into six groups; three were low-dose and three were highdose. In fact, there are many variables which might affect the actual effect of the additive such as the quality of fresh semen sample, so we managed the quality of semen as mentioned in materials and methods that depended on previous experts of our lab (Kaka et al., 2015; Khumran et al., 2015), handling of semen sample after collection until dilution and also any change in freezing temperature during the freezing process which might lead to false results. Thus, to overcome these issues, two criteria had been used; firstly, after cryopreservation we checked the viability value of one straw from the control group and the frozen sample was accepted if the control group had $50 \%$ and more viable sperms (universal standard acceptance) otherwise the sample was cancelled. The second criterion was we repeated the conventional parameters in terms of sperm motility, morphology and viability for three different times to be more precise.

Based on our findings, in chilled form, the sperm motility (Table 1), morphology (Table 2) and viability (Table 3 ) values of $T_{1}, T_{2}$, and control groups were higher than $T_{3}, T_{4}, T_{5}$ and $T_{6}$ in all evaluation periods. These findings were in contrast with our hypothesis and PGe appeared to have a negative effect on chilled semen. Sperm membrane reliability (Figure 2) had also decreased at the same manner of sperm motility (\%). Thus, these finding indicated that PGe had a detrimental effect on chilled semen, and the low concentration of PGe did not have a beneficial effect for chilled semen. In the present study the sperm motility values in groups $T_{1}, T_{2}$ and control were in line with Nair et al. (Nair et al., 2006) who reported $(71.00 \pm 3.24)$ at 0 h. However, our finding of the sperm motility (\%) was higher with that study as they reported the motility value decrease to be $(19.33 \pm 1.95)$ at day 3 of chilling in cattle bull, while in the present study the sperm motility was $(64.87 \pm 2.35)$ at day 3 in $\mathrm{T} 2$ group.

Regardless of the effect of PGe, the total sperm motility, morphology, viability, and sperm membrane reliability were significantly decreased after six days of chilling in all groups. These findings were in agreement with previous studies (Murphy et al., 2013; Nair et al., 2006).

The frozen-thawed semen showed a similar manner as in chilled semen. Since, the sperm motility (Table 4), morphology (Table 5) and viability (Table 6) values were significantly decreased in high doses of PGe groups as compared to control, $\mathrm{T}_{1}$ and $\mathrm{T}_{2}$ groups. Furthermore, PGe had a detrimental effect on sperm morphology, particularly sperm taillessness, dag-defect and decapitated-head (data not shown). In accordance to the time of evaluation of frozen-thawed semen, there were no significant differences among periods of evaluations in all groups of frozen-thawed semen.

In 1998 Chen et al. (1998) were found that, the motility of human sperm was improved when incubated with 1 and $2 \mathrm{mg} / \mathrm{mL}$ of Panax notoginseng. Our study is in agreement with a study by Gray et al. (2016) who found that, the total progressive and kinetic motilities of boar semen which was incubated with PGe $2 \mathrm{mg} / \mathrm{mL}$ did not differ from control and other groups; they attributed this to the fact that semen samples may receive insufficient amounts from ginsenosides Rc and Rb2 that is responsible for improving sperm motility (Chen et al., 1998). This was unlike Yun et al. (2016) who found that the motility of sperm treated with PGe at $2 \mathrm{mg} / \mathrm{mL}$, was greater in comparison to the control group and in line with anti-oxidative enzyme groups. They concluded that, PGe exhibited an overall positive effect on the male reproductive system of guinea pigs. However, in the present study the quality of frozenthawed bull semen was diminished in dosage of $1 \mathrm{mg} / \mathrm{mL}$ and above. This contrary finding needs further investigations to understand the mechanism of action of PGe.

The sperm plasma membranes are the main spot of destruction prompted through cryopreservation (Abavisani et al., 2013). In addition, sperm plasma membranes play an important part in the fertilization capability and spermoocyte relations (Abayasekara and Wathes, 1999). Unfortunately, sperm membrane reliability were not improved in most treatment groups and the highest value was $(58.28 \pm 2.13 \%)$ in the control group (Figure 3). Based on the results of the present study, the high dose of $\mathrm{PGe}$ had a negative effect on frozen-thawed bull semen, i.e. findings indicated that with an increase in PGe $\left(\mathrm{T}_{3}, \mathrm{~T}_{4}, \mathrm{~T}_{5}\right.$, and $\left.\mathrm{T}_{6}\right)$ resulted in poor quality of chilled and frozen-thawed 
semen. On the other hand, the low doses of PGe groups $\left(T_{1}\right.$, and $\left.T_{2}\right)$ were in line with the control group in most parameters that were evaluated in this study. Our findings are further strengthened with Kaka et al. (2016) who used tris-egg yolk diluent and Abavisani et al. (2013) and Kandelousi et al. (2013) who used citrate based diluent supplemented with poly-unsaturated fatty acids (PUFAs). These studies found that the high dose of PUFAs could reduce the quality of frozen-thawed bull semen such as in terms of sperm motility, morphology, viability, and membrane reliability. However, findings of the present study were in contrast with Tvrdá et al. (2016) in which they revealed that curcumin had protective effects on the functional activities and structural integrity of spermatozoa even when the oxidative stress increased. The present study is also in contrast with Sapanidou et al. (2015) who demonstrated that addition of $1 \mathrm{mM}$ of curcumin (active component of saffron plant) could improve the sperm motility, viability and DNA integrity of frozen-thawed bull semen.

Sperm exposure to traumatic situations of freezing-thawing may compromise the mechanism resulting in inhibited fertilization or embryonic growth (Kasimanickam et al., 2007; Khalifa et al., 2008). Kasimanickam et al. (2007) and Lymberopoulos and Khalifa (2010) showed that rising proportions of DNA-damaged sperm was related to declining field fertility of frozen-thawed bull semen. Similarly, Khalifa et al. (2008) show that, a substantial negative association exists between the occurrence of DNA-damaged sperm in frozen-thawed semen and the developing capability of bovine embryos in vitro. On the other hand, one of the main objectives of the present study was to improve the quality of sperm chromatin stability of cryopreserved bull semen. However, PGe negatively affected sperm chromatin stability (Table 7). The low dosage groups $\left(T_{1}, T_{2}\right.$ and $\left.T_{3}\right)$ which were received $(0.25 \mathrm{mg} / \mathrm{mL}, 0.5 \mathrm{mg} / \mathrm{mL}$ and $1 \mathrm{mg} / \mathrm{mL}$, respectively) from PGe were not significant as compared with the control group while high-dosage groups $\left(\mathrm{T}_{4}, \mathrm{~T}_{5}\right.$ and $\left.\mathrm{T}_{6}\right)$ which were received $(2.5 \mathrm{mg} / \mathrm{mL}, 5 \mathrm{mg} / \mathrm{mL}$ and $7.5 \mathrm{mg} / \mathrm{mL}$, respectively) from $\mathrm{PGe}$ were highly decreased spermatozoa characteristics.

\section{Conclusion}

In conclusion, addition of Panax ginseng aqueous extract into tris-egg yolk diluent did not improve chilled and frozen-thawed bull semen processes in dosages that were used in this study. Furthermore, the high doses of PGe have a negative effect on fresh, chilled and frozen-thawed bull semen. Furthermore, this study was a good opportunity to investigate this medicinal plant extract's effect directly on cell biology and function.

\section{Acknowledgments}

The authors wish to acknowledge the management and staff of University Putra Malaysia farm (TPU) and Theriogenology and Cytogenetics laboratory of the Faculty of Veterinary Medicine, UPM for their cooperation during this study. The authors also would like to thank the company L Jack $(\mathrm{M}){ }^{\circledR}$ for providing the extract of Panax ginseng with certification.

\section{Conflict of interests}

The authors declare that they have no conflict of interests.

\section{References}

Abavisani, A., Arshami, J., Naserian, A. A., Kandelousi, M. A. S., \& Azizzadeh, M. (2013). Quality of bovine chilled or frozen-thawed semen after addition of omega-3 fatty acids supplementation to extender. Int $J$ Fertil Steril, 7(3), 161.

Abayasekara, D., \& Wathes, D. (1999). Effects of altering dietary fatty acid composition on prostaglandin synthesis and fertility. Prostag Leukotr Ess, 61(5), 275-287. https://doi.org/10.1054/plef.1999.0101

Abul Rashid, B., \& Nurin Qistina, M. N. (2015). Preliminary Evaluation on the Use of Coconut Water as Semen Dilent for Local Kampung Cockerel. Paper presented at the Proc. 2nd ARCP \& 36th MASP Ann. Conf.,1-3 June 2015, Pordickson, Negeri Sembilan, Malaysia.

Amann, R. P., \& Waberski, D. (2014). Computer-assisted sperm analysis (CASA): capabilities and potential developments. Theriogenology, 81(1), 5-17. e13. https://doi.org/10.1016/j.theriogenology.2013.09.004

Amirat-Briand, L., Bencharif, D., Vera-Munoz, O., Pineau, S., Thorin, C., Destrumelle, S., . . Shmitt, E. (2010). In vivo fertility of bull semen following cryopreservation with an LDL (low density lipoprotein) extender: Preliminary results of artificial inseminations. Anim Reprod Sci, 122(3), 282-287. https://doi.org/10.1016/j.anireprosci.2010.09.006

Attele, A. S., Wu, J. A., \& Yuan, C. S. (1999). Ginseng pharmacology: multiple constituents and multiple actions. Biochem pharmacol, 58(11), 1685-1693. https://doi.org/10.1016/S0006-2952(99)00212-9

Baghshahi, H., Riasi, A., Mahdavi, A., \& Shirazi, A. (2014). Antioxidant effects of clove bud (Syzygium 
aromaticum) extract used with different extenders on ram spermatozoa during cryopreservation. Cryobiology, 69(3), 482-487. https://doi.org/10.1016/j.cryobiol.2014.10.009

Baiee, F. H., Abd Wahid H., Rosnina Y., Ariff O., Yimer, N., Zaid J., Salman H., Tarig A. A., W-N. Fitri, \& M. Umar. (2017). Kinetic Motilities of Cryopreserved Bull Spermatozoa: Owing to the Effect of Eurycoma longifolia Jack Aqueous Extract. American J Anim Vet Sci, 12(2). https://doi.org/10.3844/ajavsp.2017.77.84

Baiee, F. H., Wahid, H., Rosnina, Y., Ariff, O., Yimer, N., Jeber, Z., Hammadi, S., Tarig, A., \& Harighi, F. (2018a). Impact of Eurycoma longifolia extract on DNA integrity, lipid peroxidation, and functional parameters in chilled and cryopreserved bull sperm. Cryobiology, 80, 43-50. https://doi.org/10.1016/j.cryobiol.2017.12.006

Baiee, F., Wahid, H., Rosnina, Y., Ariff, O., \& Yimer, N. (2018b). Sperm DNA Impairment in the Bull: Causes, Influences on Reproduction and Evaluations. Pertanika J Trop Agricul Sci, 41(1).

Bollwein, H., Fuchs, I., \& Koess, C. (2008). Interrelationship between plasma membrane integrity, mitochondrial membrane potential and DNA fragmentation in cryopreserved bovine spermatozoa. Reprod Domes Anim, 43(2), 189-195. https://doi.org/10.1111/j.1439-0531.2007.00876.x

Borges-Silva, J. C., Silva, M. R., Marinho, D. B., Nogueira, E., Sampaio, D. C., Oliveira, L. O. F., \& Sartori, R. (2016). Cooled semen for fixed-time artificial insemination in beef cattle. Reprod Fert Develop, 28(7), 10041008. https://doi.org/10.1071/RD14185

Büyükleblebici, S., Tuncer, P. B., Bucak, M. N., Eken, A., Sarıözkan, S., Taşdemir, U., \& Endirlik, B. Ü. (2014). Cryopreservation of bull sperm: Effects of extender supplemented with different cryoprotectants and antioxidants on sperm motility, antioxidant capacity and fertility results. Anim Reprod Sci, 150(3), 77-83. https://doi.org/10.1016/j.anireprosci.2014.09.006

Chelucci, S., Pasciu, V., Succu, S., Addis, D., Leoni, G. G., Manca, M. E., ... Berlinguer, F. (2015). Soybean lecithin-based extender preserves spermatozoa membrane integrity and fertilizing potential during goat semen cryopreservation. $\quad$ Theriogenology, $\quad 83(6), \quad 1064-1074$. https://doi.org/10.1016/j.theriogenology.2014.12.012

Chen, J., Xu, M., Chen, L., Chen, Y., \& Chiu, T. (1998). Effect of Panax notoginseng saponins on sperm motility and progression in vitro. Phytomedicine, 5(4), 289-292. https://doi.org/10.1016/S0944-7113(98)80068-8

Cho, J.-G., Lee, M.-K., Lee, J.-W., Park, H.-J., Lee, D.-Y., Lee, Y.-H., ... Baek, N.-I. (2010). Physicochemical Characterization and NMR Assignments of Ginsenosides Rb 1, Rb 2, R c, and Rd Isolated from Panax ginseng. $J$ Ginseng Res, 34(2), 113-121. DOI:10.5142/jgr.2010.34.2.113

Eidan, S. M. (2016). Effect on post-cryopreserved semen characteristics of Holstein bulls of adding combinations of vitamin $\mathrm{C}$ and either catalase or reduced glutathione to Tris extender. Anim Reprod Sci, 167, 1-7. https://doi.org/10.1016/j.anireprosci.2016.01.014

Felipe-Pérez, Y. E., de Lourdes Juárez-Mosqueda, M., Hernández-González, E. O., \& de Jesús Valencia, J. (2008). Viability of fresh and frozen bull sperm compared by two staining techniques. Acta Vet Bras, 2(4), 123-130. https://doi.org/10.21708/avb.2008.2.4.895

Gadea, J., Gumbao, D., Canovas, S., García-Vázquez, F. A., Grullón, L. A., \& Gardón, J. C. (2008). Supplementation of the dilution medium after thawing with reduced glutathione improves function and the in vitro fertilizing ability of frozen-thawed bull spermatozoa. Int $J$ Androl, 31(1), 40-49. https://doi.org/10.1111/j.1365-2605.2007.00756.x

Galarza, D., de Guevara, M. L., Beltrán-Breña, P., Sánchez-Calabuig, M., López-Sebastián, A., Santiago-Moreno, J., \& Rizos, D. (2019). 137 Assessment of fertilizing ability of Merino ram semen cold stored up to $48 \mathrm{~h}$ by heterologous IVF of bovine oocytes. Reprod Fert Develop, 31(1), 194-194. https://doi.org/10.1071/RDv31n1Ab137

Gray, S. L., Lackey, B. R., \& Boone, W. R. (2016). Effects of Panax ginseng, zearalenol, and estradiol on sperm function. J Ginseng Res, 40(3), 251-259. https://doi.org/10.1016/j.jgr.2015.08.004

Holt, W., Del Valle, I., \& Fazeli, A. (2015). Heat shock protein A8 stabilizes the bull sperm plasma membrane during cryopreservation: Effects of breed, protein concentration, and mode of use. Theriogenology, 84(5), 693-701. https://doi.org/10.1016/j.theriogenology.2015.05.004

Kaefer, C., Komninou, E. R., Campos, V. F., de Leon, P. M., Arruda, F. V. S., Nascimento, K. S., ... Deschamps, J. C. (2013). Binding pattern and toxicological effects of lectins from genus Canavalia on bovine sperm. Reprod Toxicol, 38, 72-80. https://doi.org/10.1016/j.reprotox.2013.03.003 
Kaka, A., Haron, W., Leghari, R. A., Memon, M. I., Kaka, U., Mirani, A. H., ... Kalwar, Q. (2016). Effect of invitro supplementation of polyunsaturated fatty acids on frozen-thawed bull sperm characteristics using Bioxcell ${ }^{\circledR}$ extender. Pure Appl Biol, 5(3), 399-405. http://dx.doi.org/10.19045/bspab.2016.50052

Kaka, A., Wahid, H., Rosnina, Y., Yimer, N., Khumran, A., Behan, A. A., \& Ebrahimi, M. (2015). Alpha-Linolenic Acid Supplementation in Tris Extender Can Improve Frozen-Thawed Bull Semen Quality. Reprod Domes Anim, 50(1), 29-33. https://doi.org/10.1111/rda.12445

Kandelousi, M. S., Arshami, J., Naserian, A., \& Abavisani, A. (2013). The effects of addition of omega-3, 6, 9 fatty acids on the quality of bovine chilled and frozen-thawed sperm. Open Vet J, 3(1), 47.

Kasimanickam, R., Kasimanickam, V., Thatcher, C., Nebel, R., \& Cassell, B. (2007). Relationships among lipid peroxidation, glutathione peroxidase, superoxide dismutase, sperm parameters, and competitive index in dairy bulls. Theriogenology, 67(5), 1004-1012. https://doi.org/10.1016/j.theriogenology.2006.11.013

Khalifa, T., Rekkas, C., Lymberopoulos, A., Sioga, A., Dimitriadis, I., \& Papanikolaou, T. (2008). Factors affecting chromatin stability of bovine spermatozoa. Anim Reprod Sci, 104(2), 143-163. https://doi.org/10.1016/j.anireprosci.2007.02.019

Khoshvaght, A., Towhidi, A., Zare-shahneh, A., Noruozi, M., Zhandi, M., Davachi, N. D., \& Karimi, R. (2015). Dietary n-3 PUFAs improve fresh and post-thaw semen quality in Holstein bulls via alteration of sperm fatty acid composition. Theriogenology, 85(5), 807-812. https://doi.org/10.1016/j.theriogenology.2015.10.023

Khumran, A., Yimer, N., Rosnina, Y., Ariff, M., Wahid, H., Kaka, A., \& Sarsaifi, K. (2015). Butylated hydroxytoluene can reduce oxidative stress and improve quality of frozen-thawed bull semen processed in lecithin and egg yolk based extenders. Anim Reprod Sci, 163, 128-134. https://doi.org/10.1016/j.anireprosci.2015.10.007

Kopalli, S. R., Cha, K.-M., Jeong, M. S., Lee, S.-H., Sung, J. H., Seo, S. K., \& Kim, S. K. (2016a). Pectinasetreated Panax ginseng ameliorates hydrogen peroxide-induced oxidative stress in GC-2 sperm cells and modulates testicular gene expression in aged rats. $J$ Ginseng Res, 40(2), 185-195. https://doi.org/10.1016/j.jgr.2015.08.005

Kopalli, S. R., Won, Y. J., Hwang, S.-Y., Cha, K.-M., Kim, S. Y., Han, C. K., \& Kim, S. K. (2016b). Korean red ginseng protects against doxorubicin-induced testicular damage: An experimental study in rats. J Funct Foods, 20, 96-107. https://doi.org/10.1016/j.jff.2015.10.020

Kumar, P., Kumar, D., Sikka, P., \& Singh, P. (2015). Sericin supplementation improves semen freezability of buffalo bulls by minimizing oxidative stress during cryopreservation. Anim Reprod Sci, 152, 26-31. https://doi.org/10.1016/j.anireprosci.2014.11.015

Lymberopoulos, A., \& Khalifa, T. (2010). Sperm chromatin stability during in vitro manipulation of beef bull semen. Reprod Domes Anim, 45(2), 307-314. https://doi.org/10.1111/j.1439-0531.2008.01301.x

Medeiros, C., Forell, F., Oliveira, A., \& Rodrigues, J. (2002). Current status of sperm cryopreservation: why isn't it better? Theriogenology, 57(1), 327-344. https://doi.org/10.1016/S0093-691X(01)00674-4

Métayer, S., Dacheux, F., Dacheux, J. L., \& Gatti, J. L. (2002). Comparison, characterization, and identification of proteases and protease inhibitors in epididymal fluids of domestic mammals. Matrix metalloproteinases are major fluid gelatinases. Biol Reprod, 66(5), 1219-1229. https://doi.org/10.1095/biolreprod66.5.1219

Moustafa, M. H., Sharma, R. K., Thornton, J., Mascha, E., Abdel-Hafez, M. A., Thomas, A. J., \& Agarwal, A. (2004). Relationship between ROS production, apoptosis and DNA denaturation in spermatozoa from patients examined for infertility. Human Reprod, 19(1), 129-138. https://doi.org/10.1093/humrep/deh024

Murphy, C., Fahey, A., Shafat, A., \& Fair, S. (2013). Reducing sperm concentration is critical to limiting the oxidative stress challenge in liquid bull semen. $J$ Dairy Sci, 96(7), 4447-4454. https://doi.org/10.3168/jds.2012-6484

Nair, S. J., Brar, A., Ahuja, C., Sangha, S., \& Chaudhary, K. (2006). A comparative study on lipid peroxidation, activities of antioxidant enzymes and viability of cattle and buffalo bull spermatozoa during storage at refrigeration temperature. Anim Reprod Sci, 96(1), 21-29. https://doi.org/10.1016/j.anireprosci.2005.11.002

Papa, P. M., Maziero, R. D., Guasti, P. N., Junqueira, C. R., Freitas-Dell'Aqua, C. P., Papa, F. O., ... Dell'Aqua, J. A. (2015). Effect of glycerol on the viability and fertility of cooled bovine semen. Theriogenology, 83(1), 107-113. https://doi.org/10.1016/j.theriogenology.2014.08.009

Patel, S., \& Rauf, A. (2017). Adaptogenic herb ginseng (Panax) as medical food: Status quo and future prospects. 
Biomed Pharmacother, 85, 120-127. https://doi.org/10.1016/j.biopha.2016.11.112

Revell, S., \& Mrode, R. (1994). An osmotic resistance test for bovine semen. Anim Reprod Sci, 36(1), 77-86. https://doi.org/10.1016/0378-4320(94)90055-8

Sapanidou, V., Taitzoglou, I., Tsakmakidis, I., Kourtzelis, I., Fletouris, D., Theodoridis, A., ... Tsantarliotou, M. (2015). Antioxidant effect of crocin on bovine sperm quality and in vitro fertilization. Theriogenology, 84(8), 1273-1282. https://doi.org/10.1016/j.theriogenology.2015.07.005

Tarig, A., Wahid, H., Rosnina, Y., Yimer, N., Goh, Y., Baiee, F., Hammadi, S., \& Ebrahimi, M. (2017). Effect of different concentrations of egg yolk and virgin coconut oil in Tris-based extenders on chilled and frozenthawed bull semen. Anim Reprod Sci, 182, 21-27. https://doi.org/10.1016/j.anireprosci.2017.03.024

Tejada, R., Mitchell, J. C., Norman, A., Marik, J., \& Friedman, S. (1984). A test for the practical evaluation of male fertility by acridine orange (AO) fluorescence. Fertil steril, 42(1), 87-91. https://doi.org/10.1016/S00150282(16)47963-X

Tvrdá, E., Tušimová, E., Kováčik, A., Paál, D., Greifová, H., Abdramanov, A., \& Lukáč, N. (2016). Curcumin has protective and antioxidant properties on bull spermatozoa subjected to induced oxidative stress. Anim Reprod Sci, 172, 10-20. https://doi.org/10.1016/j.anireprosci.2016.06.008

Yimer, N., Noraisyah, A., Rosnina, Y., Wahid, H., Sarsaifi, K., \& Hafizal, A. (2014). Comparison of cryopreservative effect of different levels of omega-3 egg yolk in citrate extender on the quality of goat spermatozoa. Pakistan Vet J, 34(3), 347-350.

Yun, S. J., Bae, G. S., Park, J. H., Song, T. H., Choi, A., Ryu, B. Y., \& Chang, M. B. (2016). Antioxidant effects of cultured wild ginseng root extracts on the male reproductive function of boars and guinea pigs. Anim Reprod Sci, 170, 10. https://doi.org/10.1016/j.anireprosci.2016.04.002

\section{Copyrights}

Copyright for this article is retained by the author(s), with first publication rights granted to the journal.

This is an open-access article distributed under the terms and conditions of the Creative Commons Attribution license (http://creativecommons.org/licenses/by/4.0/). 